\title{
Approccio Sistemico Vitale e Aspect Based Received Sentiment Analysis per il governo del territorio $8^{\text {th }}$ February 2018 Revised April 2018 Accepted

Sergio Barile - Irene Fulco - Francesca Loia - Pietro Vito

\begin{abstract}
Obiettivi del paper: Il paper propone un modello concettuale di supporto al processo decisionale dell'Organo di Governo territoriale, che fornisce un quadro rappresentativo della varietà di aspettative, prospettive e interessi presenti nel contesto di riferimento, consentendo una maggiore consapevolezza degli interventi volti a risolvere le problematiche urbane.

Metodologia: Il modello proposto, basato sulla tecnica di Aspect Based Sentiment Analysis (ABSA), attraverso la lente del corpus teorico dell'Approccio Sistemico Vitale (ASV), è capace di mettere in luce i sentimenti degli attori presenti in un sistema vitale dinamico e complesso quale è il territorio.

Risultati: Il lavoro perviene allesposizione di valutazioni condivise sui livelli di "sentiment" percepiti dalla comunità rispetto ad un punto di interesse urbano considerato, guidando l'Organo di Governo territoriale nel porre in essere le condizioni per l'emergere di consonanza di contesto.

Limiti della ricerca: Le logiche delineate in una prima sperimentazione, condotta esclusivamente su un punto urbano, dovranno essere estese ad unarea d'interesse e ad un modello più sofisticato, per compiere analisi territoriali più accurate.

Implicazioni pratiche: Il modello si presenta come un valido supporto al processo di decision making territoriale, proponendo valutazioni condivise riguardo le potenzialità e le criticità latenti legate ai contesti urbani da cui trarre future linee d'azione.
\end{abstract}

Originalità del paper: Loriginalità del paper risiede nel combinare, in un unico modello, l'ASV, lente interpretativa della realtà, con l'ABSA, adattando quest'ultima al contesto territoriale.

Parole chiave: processo decisionale; approccio sistemico vitale; Aspect Based Sentiment Analysis; governo del territorio; modello di supporto alle decisioni; consonanza

\section{Viable Systems Approach and Aspect Based Sentiment Analysis for governing the territory}

Purpose of the paper: This paper proposes a conceptual model of support to the decision-making process of the territory governing body, which provides a picture representative of the variety of expectations, perspectives and interests present in the reference context, allowing for greater awareness of the interventions that could be aimed at solving urban problems.

Methodology: The proposed model, based on Aspect Based Sentiment Analysis (ABSA), aims to highlight, through the theoretical lens of the Viable Systems Approach (VSA), the sentiments of all the actors involved in a dynamic and complex viable system such as the territory. 
sinergie Vol. 37, Issue 1, 2019

Findings: The study leads to an exposition of shared evaluations on the levels of "sentiment" as perceived by the community in relation to an urban point of interest, leading the territory governing body to achieving the conditions necessary for the emergence of context consonance.

Research limits: The logics outlined through a first experimentation, carried out exclusively on a single urban point of interest, should be extended to an urban area of interest and to a more sophisticated model, aiming to perform more accurate analyses.

Practical implications: The model presents itself as a valid support system to decision making in relation to territory dynamics, proposing community-shared evaluations regarding the potentialities and latent critical flaws of urban contexts from which to define future courses of action.

Originality of the paper: The originality of the paper lies in combining, in a single model, the VSA, interpretative lens of reality, and the ABSA, adapting it to the environmental context.

Key words: decision making; viable systems approach; Aspect Based Sentiment Analysis; territory government; decision support systems; consonance

\section{Introduzione}

Le problematiche di governo del territorio e, in questo ambito, di specifici punti di interesse turistici e culturali richiedono approcci olistici che tengano conto del sistema di cui tali luoghi fanno parte e della varietà di categorie di portatori di interesse, che vanno dai fruitori agli Organi di Governo, passando attraverso gli attori che partecipano allo sviluppo del territorio.

Alla soluzione del problema di definire strategie efficaci per la conservazione e la garanzia di continuità di fruizione di tali punti di interesse possono concorrere le dottrine economico-manageriali con i loro principi. Inoltre, si sta oggi affacciando, data l'ampiezza, la profondità e la tempestività crescenti del flusso informativo e della capacità di elaborazione dello stesso, la possibilità resa dal Web di esplorare tutte le opinioni rilevanti e raccogliere tutte le informazioni necessarie, fruendone in quadri sinottici di sintesi. Questi costituiscono importanti supporti alle decisioni in quanto contribuiscono all'incremento delle varietà informative dei soggetti e, ove opportunamente gestiti, allineano progressivamente queste ultime in un processo di consonanza sistemica (Barile et al., 2015).

Gli Studiosi di economia d'impresa forniscono una ricca strumentazione sul decision making strategico. Secondo Panati e Golinelli (1988), le decisioni strategiche a) riguardano il lungo periodo e influiscono sulla sopravvivenza e sulle direzioni di sviluppo delle imprese; b) sono centralizzate, in quanto vengono assunte da un numero limitato di soggetti; c) scaturiscono in condizioni di incertezza; d) non sono ripetitive e non sono, dunque, risolvibili attraverso un confronto tra decisioni alternative o con quelle formulate in passato.

Riguardo alla genesi delle decisioni strategiche, sono ormai generalmente accettate le posizioni - tra le altre - di Panati e Golinelli (1988) e di Eisenhardt e Zbaracki (1992) che individuano la loro origine 
in un processo che contempera opportunamente razionalità (seppur limitata) del ragionamento scientifico e intuizione propria della creatività manageriale, così definendo un continuum i cui confini interni sono tracciati principalmente dalla disponibilità di dati ed informazioni e dalla capacità di elaborazione degli stessi.

Alla base del concetto di razionalità limitata di Simon (1957) ritroviamo, infatti, il postulato secondo il quale "ad eccezione di problematiche particolarmente semplici, non è ragionevole, nella generalità dei casi, attribuire ai decisori tanto la conoscenza tecnica quanto la capacità di calcolo necessarie per determinare i percorsi risolutivi previsti dalla teoria" (Barile et al., 2015).

Nel moderno ambiente informativo, in cui si vanno prepotentemente affermando interpretazioni e conclusioni basate su "big data", appare tuttavia postulabile che, nel mix tra razionalità limitata e caratteristiche di intuizione, induzione, deduzione e ragionamento logico del decisore, intervengano fenomeni osmotici intensi: da un lato, tali caratteristiche possono supporsi rafforzate dalle moderne rappresentazioni olistiche di anche grandissime quantità di dati ed informazioni e dalla possibilità crescente della loro elaborazione; dall'altro, la capacità di calcolo restituisce alla componente razionale del decision making nuova linfa e vigore (Intezari e Gressel, 2017).

Alla luce di queste considerazioni, la prospettiva olistica dell'Approccio Sistemico Vitale (ASV) (Golinelli, 2002, 2005, 2011; Barile, 2009a; Barile et al., 2015), peraltro in via di applicazione con successo in diversi ambiti di attività economica e sociale (Polese et al., 2017; Toledo et al., 2017; Walker, 2017), consente di abbracciare la complessità del problema in cui l'Organo di Governo è deputato a ricercare consonanza tra le varietà informative di tutti i sistemi interessati all'esito del suo processo decisionale. In questo quadro, la disponibilità di big data e di strumenti per la loro elaborazione incrementa la probabilità di generare risonanza. Tramite una rilettura in chiave sistemico vitale, si rileva la responsabilità di un'organizzazione nei confronti delle diverse entità con le quali si relaziona, direttamente o indirettamente (sovrasistemi e subsistemi), nella prospettiva soggettiva dell'Organo di Governo (OdG), il quale, sulla base del principio di rilevanza, agisce sulle condizioni di sopravvivenza dell'organizzazione (Barile et al., 2014).

Nell'ASV, il moderno ambiente informativo appare in grado di accelerare i processi di allineamento delle varietà informative, agendo, in particolare, tra le dimensioni e i caratteri di cui essa è costituita (Barile et al., 2015), sui livelli delle unità informative e degli schemi interpretativi di sintesi, non potendosi a priori escludere effetti di varia entità anche sugli schemi interpretativi generali e sulle categorie valoriali.

\section{Il decision making nella letteratura}

Sullo strategic decision making esistono vasti corpi letterari in vari ambiti disciplinari, tanto che Eisenhardt e Zbaracki, già nel 1992, rilevavano che "the character of the field resembles a 'crazy quilt' of perspectives". Gli
Sergio Barile

Francesca Loia Pietro Vito

Approccio sistemico vitale e aspect based sentiment analysis per il governo del territorio 
sinergie Vol. 37, Issue 1, 2019

Autori, partendo dagli studi seminali di Simon (1957), effettuano una review critica della letteratura fino ad allora esistente, identificando tre fondamentali approcci: "Rationality and Bounded Rationality", "Politics and Power", "Garbage Can", concludendo, tra l'altro, che i risultati empirici evidenziano il carattere di sistemi politici delle organizzazioni; in essi gli organi delegati alle decisioni strategiche hanno obiettivi parzialmente confliggenti e razionalità limitata (in termini di carente disponibilità di dati ed informazioni e di capacità di elaborazione degli stessi).

Alla sistematizzazione di Eisenhardt e Zbaracki (1992) sono succeduti molteplici contributi adottanti le più varie prospettive e metodologie, il che rende pretenzioso effettuare una pur breve review, per quanto narrativa o storica, della letteratura manageriale sull'argomento. A questo proposito, appare utile considerare il contributo di Shepherd e Rudd (2014), in cui la letteratura esistente al 2012 viene sistematizzata in quattro cluster sulla base delle tematiche prevalenti trattate: le caratteristiche dell'organo di governo (Top Management Team) in termini essenzialmente di varietà informativa; quelle della decisione strategica (importanza, incertezza, opportunità, minacce); quelle dell'ambiente esterno (stabilità, dinamismo, munificenza) ed infine le caratteristiche dell'impresa (struttura organizzativa, accentramento/decentramento decisionale, organicità/burocraticità). A partire dai cluster così identificati, viene di seguito esposto un sintetico aggiornamento del pensiero scientifico. Laggiornamento è stato effettuato attraverso una ricerca su Web of Science che ha restituito, nel periodo 20122018, 453 contributi nelle aree "business" e "management" in risposta alla query "strategic decision making". I cluster sono stati identificati utilizzando VosViewer di Van Eck e Waltman (2014), software sviluppato con la specifica finalità di costruire e visualizzare mappe bibliometriche distancebased. In Figura 1 è riportata la Network Visualization dei quattro cluster identificati attraverso l'analisi di accoppiamento bibliografico (bibliographic coupling/fractional counting).

Fig. 1: Bibliographic coupling dei contributi analizzati

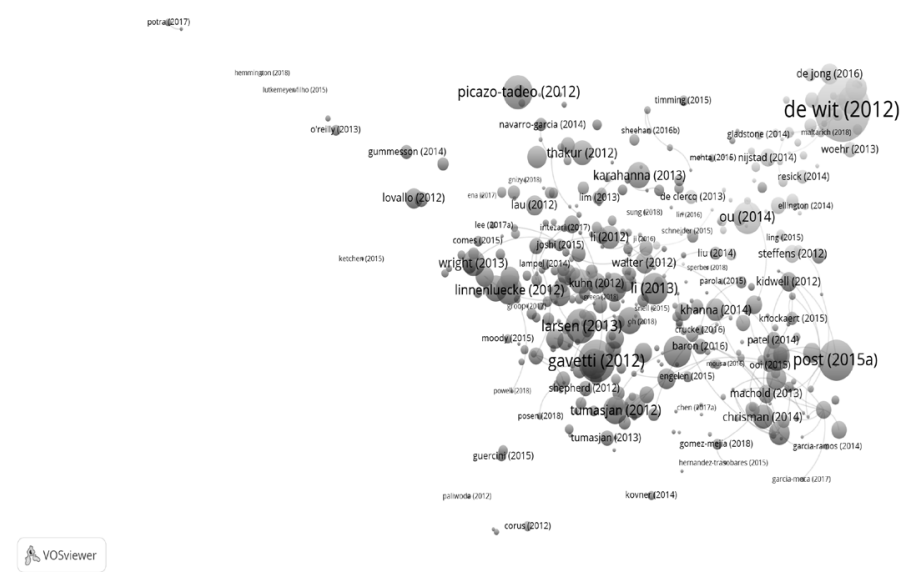

Fonte: Elaborazione degli autori tramite VOSviewer 
Nel primo cluster (De Wit et al., 2012, DeChurch, et al., 2013; De Jong et al., 2013) ricadono gli studi più numerosi ed omogenei sul tema, indicando una specifica direzione di interesse della riflessione scientifica, focalizzata sulle differenze delle varietà informative tra i componenti dellorgano di governo, i conseguenti conflitti nel processo decisionale e le ricadute sulla performance (velocità e qualità) del processo decisionale.

Nel secondo cluster (Gavetti et al., 2012; Baron et al., 2012), le caratteristiche delle decisioni vengono analizzate tramite la lente della teoria comportamentale, esplorandone i potenziali benefici e i potenziali costi (talvolta "nascosti").

I contributi del terzo cluster (Karahanna e Preston, 2013; Li et al., 2012) risultano generalmente volti ad identificare il mix tra razionalità e comportamento politico nel processo decisionale, in funzione delle caratteristiche ambientali esterne.

Nel quarto cluster (Post e Byron, 2014; Post et al., 2015, Ben-Amar et al., 2013), infine, vengono messi in relazione i temi del livello di accentramento del potere, della composizione dell'organo di governo delle imprese (board composition) e della sua dimensione con l'efficienza del processo decisionale in termini di performance e sostenibilità.

Al fine di meglio definire l'ambito di riferimento del seguente lavoro, si procede nel seguito ad una breve review dei principali contributi sullo specifico tema del decision making in ambito territoriale e del territorio inteso come sistema complesso.

\section{Il decision making nei sistemi territoriali}

\subsection{Approcci decisionali nel territorio come sistema complesso}

A partire dai contributi seminali di Calabi e Indovina (1973) e Preteceille (1974), i quali definivano il territorio come uno spazio naturale "messo in scena" nel tempo dagli esseri umani, si muovono gli studi da parte della Comunità scientifica sulla definizione di "territorio". Successivamente, il territorio è stato considerato un prodotto (Lefebvre, 1974), una struttura (Foucault, 1994), ovvero un laboratorio sociale con una specifica localizzazione geografica in cui natura ed esseri umani interagiscono nel tempo. Crosta (2000) giunge alla seguente concettualizzazione di territorio: esso è uno spazio fisico specifico, esito eventuale dell'interazione sociale, caratterizzato da un insieme di processi eterogenei (quali lo sfruttamento delle risorse naturali, la negoziazione dei conflitti e la risoluzione dei problemi). In conclusione, l'interazione tra spazio geografico, tempo e relazione sociale porta all'emersione del territorio.

Nel seguente lavoro, in particolare, e come vedremo nel paragrafo successivo, il territorio è considerato come un sistema complesso situato in uno specifico spazio geografico che emerge dalla co-evoluzione di un insieme di processi eterogenei (antropologico-culturale, relazionale, cognitivo ed economico-produttivo) (Simone et al., 2018).

La difficoltà di individuare modelli di gestione del territorio come sistema complesso, in grado di esplicitarne le potenzialità in termini di
Sergio Barile

Irene Fulco

Francesca Loia

Pietro Vito

Approccio sistemico vitale

e aspect based sentiment

analysis per il governo del territorio 
sinergie Vol. 37, Issue 1, 2019

fruibilità (attrattività turistica, benessere dei residenti, sostenibilità), riconosciuta da Ehlinger et al. (2007), viene generalmente affrontata attraverso la proposta di nuovi modelli di gestione volti a garantire efficacia, efficienza, sostenibilità per supportare le decisioni degli organi di governo. Altri contributi sono dedicati ad aspetti specifici relativi al governo dei sottosistemi esistenti nel territorio.

Tra le proposte di nuovi modelli di gestione si rileva l'attenzione verso le partnership pubblico-privato (PPP), delle quali esistono varie esperienze, alcune in Italia. Argiolas et al. (2009) valutano, sulla base di un indagine empirica su 63 Local Action Group (LAG), l'impatto di tali tipi di partnership sul governo territoriale, evidenziando che esse possono costituire un' alternativa valida ai tradizionali modelli di gestione. Velotti et al. (2012), con riferimento ad alcune città italiane, ne esplorano l'efficacia e la sostenibilità, in funzione dei livelli di legittimazione, trasparenza e responsabilità che esse detengono. L'esperienza di partecipazione di comitati locali alle decisioni urbane relative ad alcune importanti città francesi viene valutata, in una prospettiva storica, da Mattina (2008), il quale si focalizza, in particolare, sui conflitti insorti nel tempo tra i rappresentanti delle organizzazioni intermedie e gli organi di governo del territorio.

Le potenzialità del crowdsourcing come fonte di conoscenze ed informazioni per il supporto alle decisioni vengono esplorate da Certoma et al. (2015), che suggeriscono ai decisori di dedicare maggiori risorse alle apposite piattaforme. Il quadro teorico di fondo risale alla teoria sociologica della "saggezza della folla" (Galton, 1907; Surowiecki, 2007), che sta oggi influenzando significativamente i fenomeni sociali per le possibilità di comunicazione ed aggregazione offerte dai social software.

Il tema del governo del sottosistema dei trasporti negli agglomerati urbani viene trattato da Ciasullo et al. (2016), con riferimento alla mobilità dei passeggeri, e da Samuel e Carré (2018), relativamente alla movimentazione delle merci. In particolare, Ciasullo et al. (2016) si focalizzano, con riferimento allarea urbana di Bologna, sulla possibilità di ricombinazione delle risorse esistenti offerta dalle soluzioni ICT nellottica di co-creazione di valore in un sistema di servizi. Samuel e Carré (2018) percepiscono, invece, l'insorgere di comunità, definite dagli stessi "ibride", data lintensificazione delle relazioni tra soggetti pubblici e privati finalizzate ad affrontare le problematiche ambientali e sociali poste dalla logistica urbana, in relazione alla movimentazione ed alla distribuzione delle merci.

Per quanto concerne gli studi relativi agli ambienti dinamici e complessi, risultano essere di particolare rilevanza anche i contributi di Crouch e Ritchie (1999), Buhalis (2000) e Tamma (2000), i quali si soffermano sullo strategic management delle nuove configurazioni territoriali. In ottica sistemica, gli avanzamenti sul tema si sono focalizzati sui sistemi di offerta turistici (Della Corte, 2000), sugli ecosistemi (Pilotti e Tedeschi-Toschi, 2011; Pilotti et al., 2013), sulla competitività delle destinazioni turistiche (Martini, 2002; Franch, 2002; Pencarelli e Forlani, 2003) e sulle risposte strategiche alla complessità (Rispoli e Tamma, 1995). 
Dai contributi esaminati, emerge, però, la necessità che le decisioni relative ad un sistema complesso quale il territorio debbano essere prese in modo partecipato da tutti gli attori, effettivi e potenziali, che detengono, in modo diretto o indiretto, interesse alla fruibilità del territorio stesso: ciò conduce alle riflessioni sull'adattamento reciproco degli attori e sulle condizioni di consonanza in grado di conferire alle decisioni i ricercati caratteri di efficacia, efficienza e sostenibilità.

Data la frammentazione dei contributi considerati e di cui sopra si è effettuato un sommario aggiornamento, emerge la necessità di una visione olistica sul tema. In questa direzione si pone la visione sistemica di Beer (1972), progressivamente arricchita da nuove concettualizzazioni da parte di Golinelli $(2005,2011)$ e Barile (2009a; Barile et al., 2015) sul decision making in ambito complesso, che abbraccia gran parte del dibattito scientifico. In particolare, al fine di pervenire a decisioni efficienti ed efficaci da parte dell'Organo di Governo, emerge la necessità di allineamento delle "varietà informative" dei diversi sistemi presenti nel territorio e si manifesta la centralità dei concetti di consonanza, risonanza e rilevanza.

\subsection{L'ASV come strumento di supporto alle decisioni nei sistemi territoriali}

Il territorio, originariamente definito tramite i concetti di area, spazio e regione e descritto, sulla base della sua configurazione fisica, come un insieme di "risorse di dotazione" (Barile e Saviano, 2008, 2011), viene interpretato, da una letteratura più recente (Barile e Golinelli, 2008; Barile, 2011; Barile e Di Nauta, 2011; Barile e Saviano, 2012; Barile et al., 2012a), come un "giacimento di risorse ed entità in azione per l'emersione di un sistema unitario" (Barile et al., 2013a). Larea geografica territoriale a cui facciamo riferimento, dunque, grazie alla lente del corpus teorico dell'ASV, non assume più una connotazione puramente strutturale, ma diviene il risultato di interazioni (relazioni attivate tra individuo e individuo e tra individuo e ambiente) che evolvono dinamicamente e si sviluppano ricorsivamente. Alle dotazioni naturali, artistiche e strutturali, incluse 'oggettivamente' nella definizione di territorio, si affiancano, quindi, le componenti sistemiche (individui, organizzazioni e istituzioni), che, con le loro visioni e proiezioni di aspettative irriducibilmente soggettive, possono perseguire finalità non condivise e non allineate al fine di sopravvivere nel contesto di riferimento (Barile e Golinelli, 2008; Barile, 2011).

Un sistema può essere definito vitale se è in grado di sopravvivere nel suo contesto di riferimento (Barile et al., 2015); questa capacità di sopravvivenza deriva dall'abilità dell'Organo di Governo di stabilire relazioni sinergiche (definite dal concetto di consonanza) con le entità che popolano quello stesso contesto. Risulta necessario, dunque, per l'Organo di Governo, fondare le decisioni su una logica partecipativa, che possa fungere da guida verso un obiettivo condiviso, basato su unarmonia di fini (consonanza). Ponendo in essere iniziative realizzate nell'interesse del territorio stesso e adottando, dunque, una visione unitaria (effetto di risonanza), si fortifica la probabilità di un potenziale passaggio da uno spinto individualismo all'emersione di una coscienza collettiva (Barile et al., 2013a; Schillaci e Gatti, 2010). 
sinergie Vol. 37, Issue 1, 2019

Il processo di contestualizzazione che conduce allestrazione, da parte dell'Organo di Governo, di uno specifico contesto di riferimento da un ambiente più generico (Golinelli, 2002, 2005, 2011; Barile, 2009a), qualifica, come illustrato in Figura 2, uno dei momenti centrali nel superamento dei limiti propri di una prospettiva meramente strutturale. In tal modo, tramite levanescenza dei confini territoriali, viene spostato il focus verso le dinamiche sistemiche e verso un bacino più ampio di relazioni inter ed intra-sistemiche che caratterizzano il territorio, accrescendo la probabilità di sopravvivenza del sistema stesso.

Fig.2: Il processo di contestualizzazione

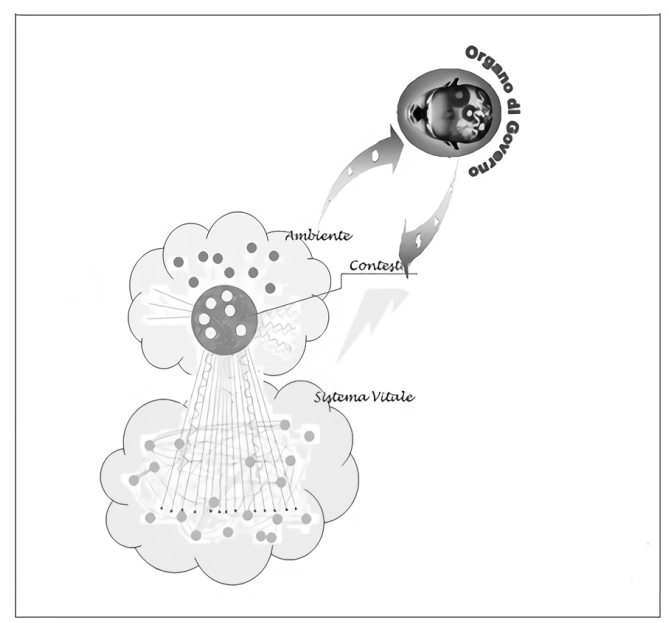

Fonte: Barile, 2010, www.ASVSA.org. Figura utilizzata con il permesso di ASVSA

Il processo di contestualizzazione pone enfasi su uno dei concetti chiave dell'ASV: il principio di rilevanza. La qualificazione del contesto avviene, infatti, sulla base del grado di interesse (in termini di criticità delle risorse e potere di influenza esercitabile) suscitato da un certo sovrasistema (individui, imprese, organizzazioni, ecc.) sull'Organo di Governo del sistema vitale preso in considerazione (Golinelli 2005; Barile e Golinelli, 2008); nel nostro caso sull'Organo di Governo multi-soggettivo e di natura composita di cui è dotato il territorio (Barile e Golinelli, 2008; Barile et al., 2018).

In sintesi, col passaggio da ambiente a contesto, l'Organo di Governo territoriale procede alla valutazione e alla selezione delle entità con cui intende interagire e, di conseguenza, alla mappatura della rete di relazioni da instaurare con le stesse (Polese e Minguzzi, 2009), allattivazione di queste relazioni (Polese, 2002) e alla definizione di una strategia che possa interpretare le loro esigenze, tradurle in obiettivi e consentirne il loro raggiungimento.

Alla luce di queste breve considerazioni, possiamo affermare che il governo del territorio, riconcepito in ottica sistemica, ci indirizza verso una nuova visione di concepire il processo decisionale: parliamo di decision making per il territorio (Barile, 2011), superando la precedente visione di 
orientamento delle decisioni e delle azioni alla creazione di valore nel territorio e del territorio.

Quanto fin qui descritto è origine, d'altro canto, dei tipici problemi legati al governo del territorio, soprattutto quando i livelli di complessità risultano essere particolarmente significativi. Il processo di valorizzazione territoriale richiede di concepire soluzioni condivise per le complesse questioni ambientali, sociali ed economiche a livello locale (Napolitano, 2000; Sciarelli, 2007; Marino et al., 2013; Carrubbo, 2013; Iandolo et al., 2016; Basile et al., 2016; Simone et al., 2018; Iandolo et al., 2019). In particolare, Basile et al. (2016) e Simone et al. (2018) inquadrano la natura complessa del governo del territorio e delle relative problematiche di decision making, proponendo, rispettivamente, uno schema interpretativo dei fenomeni di adattamento tra gli attori del territorio basato sulla teoria sistemica della complessità ed un modello in grado di supportare i decisori di fronte alla crescente sfida di una gestione del territorio efficace, efficiente e sostenibile (ConsulCubo VSA). Anche Fotino et al. (2018) adottano la lente interpretativa del VSA, facendo emergere come attraverso la stessa, lorgano di governo sia in grado di definire sistemi specifici di decisioni che rappresentano una sintesi generale delle aspettative di tutti gli attori organizzati all'interno di unarea specifica, identificando i modelli di sviluppo sui quali essi concordano.

In tal senso, la complessità può riassumersi nell'impossibilità di circostanziare il problema servendosi di schemi interpretativi, e quindi modelli, tecniche e strumenti, utilizzati nel passato.

L'avvento, inoltre, di una crescente interdipendenza tra globale e locale ha posto l'enfasi sui concetti di "relazione" e "sistema", tanto da condurre Scott e Storper (2003) a definire le nuove configurazioni territoriali come sistemi sinergici di risorse fisiche e relazionali.

È necessario, in sintesi, andare alla ricerca di soluzioni che possano essere di supporto al processo decisionale dell'Organo di Governo territoriale, che si trova a rapportarsi con numerosi e mutevoli entità del contesto in condizioni di complessità, nel tentativo di armonizzare gli interessi dei vari attori e di convergere verso una direzione evolutiva congiunta, verso una ricercata consonanza di contesto (Barile e Calabrese, 2011).

\section{Metodologia di indagine}

\subsection{Obiettivi e metodologia di ricerca}

Alla luce del quadro sopra delineato, considerando, dunque, il territorio come un sistema dinamico di relazioni intersoggettive (Barile et al., 2013a), è stato condotto uno studio, basato sulle tecniche di Sentiment Analysis, al fine di definire un modello di supporto alle decisioni dell'Organo di Governo territoriale, che metta in luce i sentimenti e le opinioni espressi all'interno di testi generati in rete (big data) (Manyika et al., 2011; Provost e Fawcett, 2013; Troisi et al., 2018) dai cittadini e dai visitatori di una specifica area territoriale, in riferimento alle risorse, alle 
sinergie Vol. 37, Issue 1, 2019

strutture o ai servizi ivi presenti. In particolare, l'idea alla base del modello è quella di applicare la tecnica di Aspect Based Sentiment Analisys (Liu, 2012), evoluzione della Sentiment Analysis (Wilson et al., 2005; Pang e Lee, 2008; Pak e Paroubek, 2010), non più per condurre analisi di mercato, come di consuetudine, bensì in un contesto urbano, al fine di rendere l'Organo di Governo, grazie alla possibilità di ottenere in output analisi aggregate a diversi livelli di granularità, consapevole delle sensazioni condivise riguardo ai principali aspetti delle risorse urbane (monumenti, luoghi di interesse turistico, servizi, ecc.). L'intero sistema proposto, basato su tale modello, alla luce dell'Approccio Sistemico Vitale (ASV), consente di enfatizzare, attraverso il concetto di consonanza e risonanza territoriale, come il passaggio dallorientamento individuale a quello sistemico, che tiene conto della collettività, possa favorire un miglioramento dei processi decisionali e il conseguente convergere verso una visione unitaria.

In questa direzione, il modello di supporto alle decisioni territoriali proposto, basato, come già detto, sulle tecniche di ABSA, insieme ad una prima sperimentazione condotta su un punto di interesse, farà emergere le opinioni collettive legate allo stesso e consentirà di implementare azioni a più macrolivelli decisionali:

- valorizzare le componenti sistemiche presenti nel punto di interesse, coinvolgendole direttamente e indirettamente nello sviluppo dello specifico sistema territoriale, al fine di accrescere le probabilità di sopravvivenza del sistema stesso;

- favorire il coinvolgimento e la partecipazione tramite un tentativo di armonizzazione e allineamento delle diverse proiezioni;

- coordinare i comportamenti delle componenti coinvolte nello sviluppo dello specifico sistema territoriale;

- orientare il processo decisionale ad interventi volti a porre in essere le condizioni per l'emergere di consonanza di contesto.

\subsubsection{La ABSA applicata al sistema territoriale in ottica ASV}

L'idea è quella di applicare la tecnica di Aspect Based Sentiment Analisys, al fine di valutare le opinioni della comunità rispetto alle varie componenti degli asset urbani. La tecnica dell'Aspect Based Sentiment Analysis fornisce informazioni non più sul livello di "Sentiment" del prodotto nel suo insieme, ma circa le varie componenti dello stesso, consentendo unanalisi più precisa e puntuale (Liu, 2012; Pavlopoulos, 2014).

Nel caso specifico, si adotta la tecnica dell'ABSA, precedentemente utilizzata, come già affermato, nell'analisi dei prodotti commerciali, alle diverse componenti degli asset urbani, per rendere consapevole l'Organo di Governo territoriale delle opinioni collettive riguardo ai differenti aspetti caratterizzanti un punto di interesse.

Dunque, secondo l'approccio di Liu, la tecnica di Aspect Based Sentiment Analisys individua le sensazioni espresse dagli utenti della community oggetto di analisi, detti "holders", in riferimento ai singoli "aspects" delle risorse urbane (monumenti, luoghi di interesse turistico, servizi, ecc.), e stabilisce se esse siano positive o negative, con possibilità di analisi aggregate a diversi livelli di granularità. A tal fine definiamo: l'opinion-city come una 
quintupla $\left(e_{i}, a_{i j}, s_{i j k p}, h_{k}, t_{l}\right)$, dove ei è il nome dell'entity-city (ad esempio un monumento, una piazza, un punto di interesse turistico, ecc.), $a_{i j}$, è un aspect-city di $e_{i}$ (ad esempio con riferimento alle grandi fontane: vasca, statua, piazza), $s_{i j k l}$ è il sentiment sull'aspetto $a_{i j}$ dell'entità, $e_{i}$ (ad esempio grazioso), $h_{k}$ è l'opinion holder (soggetto che esprime il sentiment) e $t_{l}$ è il tempo in cui l'opinione è espressa da $h_{k}$. Si ottiene, dunque, una sintesi organizzata in diversi livelli di "sentiment" riguardanti le entità urbane e i diversi aspetti delle stesse, grazie al processo di trasformazione di dati non strutturati in dati strutturati. In tal modo, l'Organo di Governo territoriale può avvalersi di tale output per compiere analisi sia qualitative che quantitative, al fine di valutare in toto le opinioni degli attori individuati come rilevanti (nel caso specifico, la community di riferimento), e, quindi, accrescere la possibilità di sopravvivenza del sistema, grazie ad una ricercata consonanza.

\subsubsection{Il processo decisionale territoriale basato sulla ABSA in ottica ASV}

- Rappresentazione delle quintuple dellopinion (Liu B., 2012): è possibile generare le quintuple;

- Rappresentazione del Sentiment delle sentiment words elo expressions: utilizzando Sentiwordnet (Baccianella et al., 2010) si rappresentano i valori di "sentiment" di ogni tupla;

- Aggregazione dei risultati: si utilizza la media come funzione di aggregazione;

- Rappresentazione grafica dei risultati: mediante un grafico a barre si rappresentano sinteticamente le polarità di ogni entità della città analizzata e degli aspect individuati;

- Attività decisionale dell'organo di governo territoriale: l'Organo di Governo potrà porre in essere interventi volti a creare le condizioni per l'emergere di consonanza di contesto.

Fig. 3: Processo decisionale territoriale con tecniche di ABSA in ottica ASV

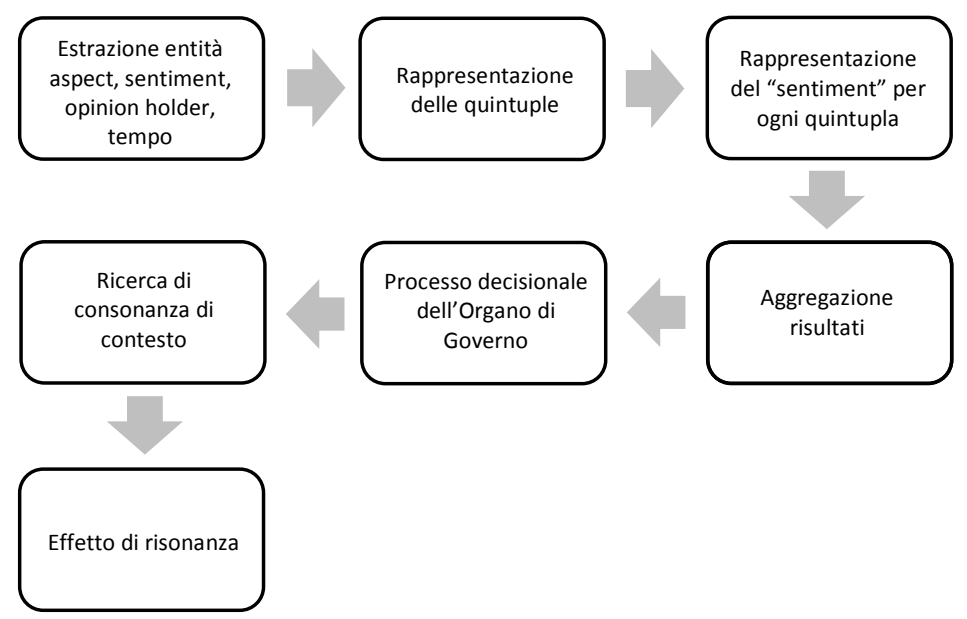

Fonte: elaborazione degli autori
Sergio Barile

Irene Fulco

Francesca Lo

Approccio sistemico vitale

e aspect based sentiment

analysis per il governo del territorio 


\section{sinergie}

Vol. 37, Issue 1, 2019

\subsection{Definizione della Community e raccolta dei dati}

È stato condotto uno studio di analisi del testo su larga scala, con lobiettivo di comprendere, relativamente ad un punto di interesse individuato, i principali sentimenti espressi all'interno di testi generati in rete dai cittadini e dai visitatori della città di Roma. Il fine è quello di definire un modello che, considerando le interazioni dinamiche tra gli attori coinvolti, supporti l'Organo di Governo territoriale di fronte alla crescente sfida di una gestione efficace ed efficiente della capitale.

La raccolta delle opinioni degli utenti avviene in una community di un Sito Web. In particolare, la scelta è ricaduta sul sito TripAdvisor.com, un portale web di viaggi che pubblica le recensioni degli utenti riguardo hotel, B\&B, appartamenti, ristoranti e attrazioni turistiche, che, con oltre 60 milioni di recensioni e opinioni, rappresenta il più grande sito di viaggi al mondo, capace di connettere dinamicamente unampia rete di relazioni.

Per il caso di studio, è di interesse soffermarsi sul punto verso il quale la popolazione nutre particolari opinioni che possono contribuire al processo decisionale dell'Organo di Governo, facendo emergere delle problematiche latenti legate alla specifica area territoriale. Nello specifico, è stato selezionato sul Sito Web uno dei punti di interesse di maggiore rilevanza per la comunità: Fontana di Trevi. Tale punto di interesse locale figura tra i primi nella classifica consigliata dal Sito Web e gode di uno dei più elevati gradi di attrattività nella città di Roma.

In particolare, l'intero corpus di recensioni sulla fontana di Trevi presente su Tripadvisor.com risulta essere, alla data della rilevazione dei dati, di 75.974 recensioni visibili. Su tale popolazione di recensioni (75.974), per ovvi vincoli di tempo e costi, individuiamo una numerosità campionaria $n$ tale da consentirci di garantire, rispetto all'intera popolazione, un errore campionario del $5 \%$ ad un livello di probabilità del 95\%. Dunque, sulla base di tali parametri, la numerosità campionaria calcolata è pari a 382 recensioni. Dall'intero corpus, quindi, attraverso un campionamento casuale semplice, vengono selezionate ed estratte 382 recensioni, opportunamente memorizzate in un repository delle recensioni che funge da alimentatore e da input per la successiva fase di analisi prevista dall'approccio. Dalle sperimentazioni effettuate risulta peraltro evidente che, a valle di una attività di integrazione, ottimizzazione e tuning delle diverse fasi, tecniche e tecnologie abilitanti, sarà possibile applicare, qualora si ritenga opportuno, il processo definito sull'intera popolazione di recensioni, praticamente a parità di risorse disponibili.

Ciò premesso, durante la fase di raccolta dei dati, utilizzando appositi tool di analisi sintattica e di knowledge extraction (D'Aniello et al., 2018), si identificano le componenti del punto di interesse a cui la community presta maggiore attenzione e gli attributi ad esse associati su cui, successivamente, attraverso la risorsa lessicale SentiWord.net, verrà applicata la tecnica di Aspect Based Sentiment Analysis e, dunque, calcolato il valore di "sentiment" percepito dalla comunità. 
Fontana di Trevi

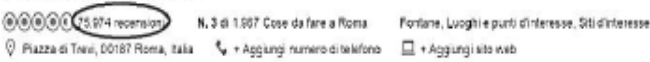
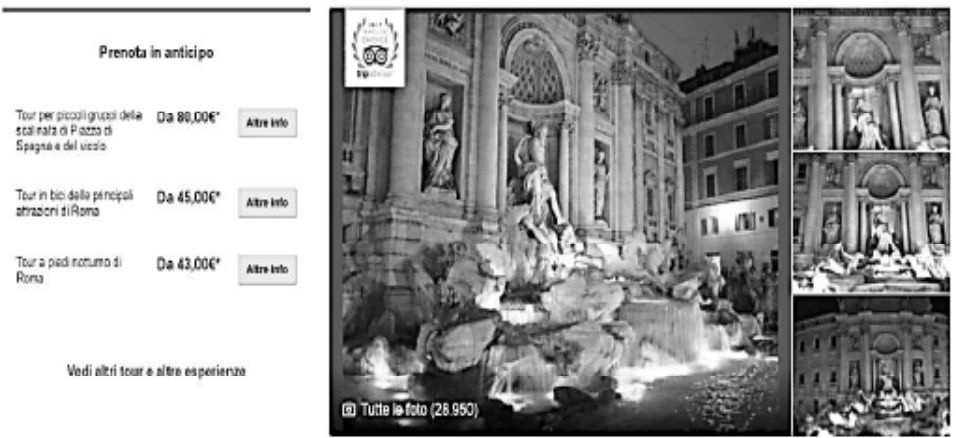

Vedi atri tour o steve esperiente

Fonte: https://www.tripadvisor.it/Attraction_Review -g187791-d190131-Review s-Trevi_ Fountain-Rome_Lazio.html, febbraio 2018.

\subsection{Analisi dei dati}

Terminata la fase di raccolta dei dati, si procede con l'estrazione dellentità e degli aspect per ciascun opinion holder che ha rilasciato una recensione in riferimento al punto di interesse in esame. Successivamente, le entità, gli aspect, le opinioni, gli opinion leader, insieme all'intervallo temporale in cui la recensione è stata lasciata, vengono rappresentati in quintuple, insiemi di cinque elementi che sono in relazione con alcuni attributi del testo estratto.

Si riportano, di seguito, alcune tuple estratte dalle recensioni rilasciate dagli utenti riguardo "Fontana di Trevi" su TripAdvisor.com:

“ $\{$ Fontana di Trevi, vasca, meravigliosa, FedericoLeoni, Sept-15-2017\};

\{Fontana di Trevi, statue, stupefacenti, Oliviero98, Nov-18-2017\};

\{Fontana di Trevi, piazza, sporca, Sandra23, Nov-21-2017\};

\{Fontana di Trevi, GENERAL, unica, MarcoForti_, Dic-3-2017\};

\{Fontana di Trevi, piazza, ampia, Carlagrami, Dic-15-2017\}”.

Successivamente, utilizzando Sentiwordnet (Esuli e Sebastiani, 2007; Denecke, 2008), si rappresentano i valori di “sentiment" di ogni tupla. In particolare, considerando ogni parola chiave estratta, i termini vengono analizzati al fine di stabilire la loro connotazione positiva, negativa o oggettiva. Il tool semantico, dunque, fornisce in output, per ciascun termine, i valori nell'intervallo $[0,1]$, che rappresentano la positività, la negatività o la neutralità di ogni parola chiave analizzata, la cui somma totale deve essere pari a 1 .

In base a quanto detto, per ciascun termine, si valutano i relativi valori di positività $S_{p i}$, negatività $S_{n i}$ o neutralità $S_{u i}$ come segue: 


\section{sinergie}

Vol. 37, Issue 1, 2019

$$
\begin{gathered}
S p_{i}=\frac{\sum_{k=1}^{K} p_{k}}{K} \\
S n_{i}=\frac{\sum_{k=1}^{K} n_{k}}{K} \\
S u_{i}=\frac{\sum_{k=1}^{K} u_{k}}{K}
\end{gathered}
$$

A titolo esemplificativo, si riportano i valori di sentiment riguardanti "Fontana di Trevi" di alcune tuple estratte:

“ $\{$ Fontana di Trevi, vasca, $(0.80,0.20,0)$, FedericoLeoni, Sept-15-2017\};

\{Fontana di Trevi, statue, $(0.85,0.15,0)$, Oliviero98, Nov-18-2017\};

\{Fontana di Trevi, piazza, (0, 0.30, 0.70), Sandra23, Nov-21-2017\};

\{Fontana di Trevi, GENERAL, (0.70, 0.30,0), MarcoForti_,Dic-3-2017\};

\{Fontana di Trevi, piazza, (0.90, 0.10, 0), Carlagrami, Dic-15-2017\}”.

Si procede, infine, con l'aggregazione dei risultati mediante la funzione media, al fine di poter compiere valutazioni a diversi livelli di granularità, soffermandosi sia sul punto di interesse nella sua totalità che sui differenti aspect che lo compongono.

\section{Risultati}

Rappresentati i livelli di "sentiment" di ogni tupla, riguardanti l'entità presa in esame e i corrispettivi aspect, si procede con l'aggregazione di questi ultimi, al fine di poter effettuare, dunque, delle valutazioni generali sul punto di interesse considerato. Dalla Tab.1 e dal grafico emerso, risulta che le opinioni collettive riguardanti "Fontana di Trevi" sono prevalentemente positive, confermando il luogo come uno dei punti della città con la più alta attrattività.

Tab. 1: Livello di Sentiment "Fontana di Trevi"

\begin{tabular}{|l|l|}
\hline & Livello di Sentiment \\
\hline Fontana di Trevi & $0.65 ; 0.05 ; 0.30$ \\
\hline
\end{tabular}

Fonte: elaborazione degli autori.

Fig. 5: Livello di "sentiment" del punto di interesse

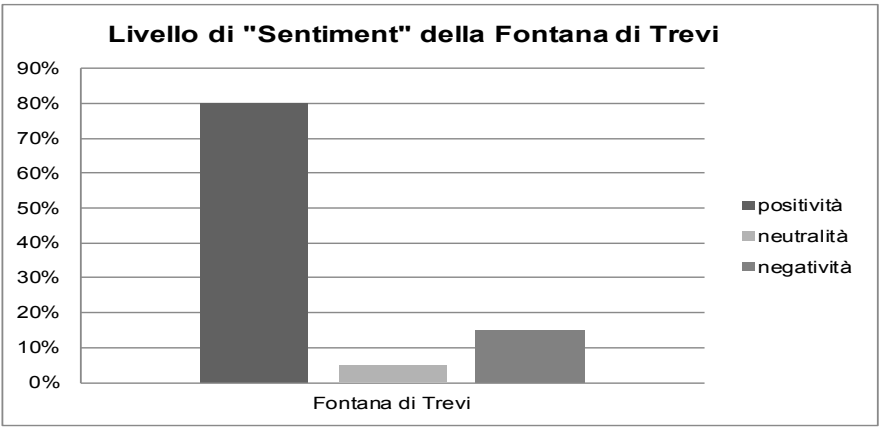

Fonte: elaborazione degli autori 
A questo punto, l'Organo di Governo territoriale può compiere $\begin{gathered}\text { Sergio Barile } \\ \text { Irene Fulco }\end{gathered}$ un'analisi più accurata, al fine di comprendere, analizzando le diversi Prancesca Loia parti che compongono il punto di interesse, se, da parte degli utenti $\begin{aligned} & \text { Pietro Vito } \\ & \text { Aproccio sistemico vitale }\end{aligned}$ della community, vi è l'insorgere di particolari sentimenti positivi, 0 , enalysis per il governo del contrariamente, opinioni negative, rappresentative di problematiche celate del contesto urbano.

Tab. 2: Valori di polarità di entità e aspect

\begin{tabular}{|c|c|c|}
\hline Entità & Aspect & Valori di Polarità \\
\hline Fontana di Trevi & & $0.65 ; 0.05 ; 0.30$ \\
\hline & GENERAL-Fontana di Trevi & $0.84 ; 0.06 ; 0.10$ \\
\hline & Vasca & $0.91 ; 0.02 ; 0.07$ \\
\hline & Piazza & $0.21 ; 0.06 ; 0.73$ \\
\hline & Statue & $0.86 ; 0.12 ; 0.2$ \\
\hline & Scalinate & $0.43 ; 0.16 ; 0.41$ \\
\hline
\end{tabular}

Fonte: elaborazione degli autori

Fig. 6: Livello di "sentiment" degli aspect

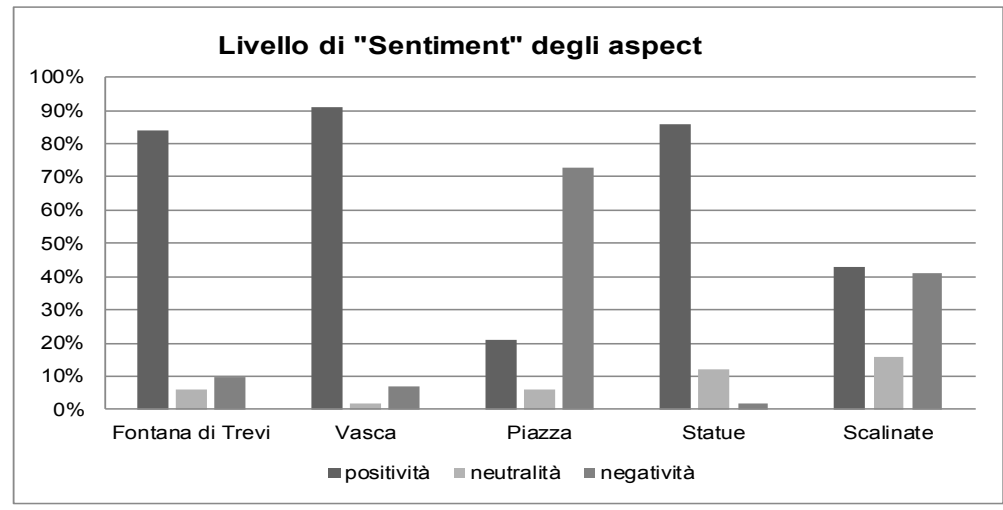

Fonte: elaborazione degli autori

Si analizza, dunque, il grafico dei risultati ottenuti che, in maniera sintetica, rappresenta le polarità di ogni aspect dell'entità analizzata. In particolare, come si può notare dalla Tab. 3 e dal grafico in Fig. 6, vi sono alcuni aspect percepiti come particolarmente positivi. Infatti, soprattutto verso gli aspect "statua" e "vasca", gli utenti hanno espresso un'opinione fortemente positiva, legata alla magnificenza delle statue, delle rocce e delle tre vasche di cui è composta la fontana. La connotazione positiva caratterizzante gli aspect suggerisce all'Organo di Governo territoriale, ad esempio, la possibilità di enfatizzare, mediante strategie di marketing, la storicità del luogo e l'attrattività legata all'aspetto della tradizione storicoculturale italiana.

Da un'analisi ancora più accurata, si evidenzia che, seppur in misura ridotta, sono presenti dei primi sintomi di malcontento da parte della 
sinergie

Vol. 37, Issue 1, 2019

community, in riferimento a particolari aspetti della Fontana di Trevi. Tali opinioni negative fanno emergere segnali velati a cui l'Organo di Governo deve necessariamente porre attenzione. Nellanalisi, infatti, il livello di "sentiment" associato all'aspect "piazza" è prevalentemente negativo, poiché, andando a considerare le tuple costruite, è emersa più volte la connessione con l'aggettivo "sporco". Tale informazione può essere di particolare rilevanza per l'Organo di Governo, che, essendo consapevole di questa problematica percepita dalla collettività, potrà destinare con maggiore capillarità le risorse dedite allo smaltimento dei rifiuti nell'aerea interessata e in quella limitrofa. Ancora, analizzando le tuple, l'aspect "piazza" risulta connesso all'aggettivo "pericoloso", sintomo di un principio di percezione fortemente negativa da parte degli utenti rispetto al luogo d'interesse, legato probabilmente alla presenza di borseggiatori nellarea vicina a "Fontana di Trevi". L'Organo di Governo dovrà, affinché tale percezione non venga condivisa da una fetta di utenti sempre maggiore e il luogo d'interesse non venga più percepito come storico e ricreativo, prendere provvedimenti di gestione delle risorse da destinare alla sicurezza del luogo.

\section{Discussione}

A partire dai risultati ottenuti, il lavoro evidenzia come, attraverso l'utilizzo della tecnica di Aspect Based Sentiment Analysis e della lente interpretativa dell'ASV, l'Organo di Governo territoriale possa diventare consapevole delle sensazioni condivise sui principali aspetti che compongono il punto di interesse Fontana di Trevi. È possibile, dunque, confrontando i quadri sintetici ottenuti, ragionare sui diversi livelli di sentiment espressi dalla community presa in esame, valutando sia le principali attrattività che le latenti criticità di unarea urbana.

Come riportato nel Paragrafo 3.1, nel contesto territoriale generalmente le iniziative sono guidate da una logica miope, che trascura le aspettative dei relativi sovrasistemi, compromettendo le probabilità di sopravvivenza a lungo termine. La problematica, dunque, risiede nel non riuscire a riconoscere l'effettiva e potenziale rilevanza degli attori di contesto (Barile et al., 2013a). Sulla base di tali elementi è possibile giungere alla caratterizzazione di un modello ricorsivo, di cui è proposta una sintetica rappresentazione in Figura 7, orientato al raggiungimento di una valorizzazione territoriale, al fine di sviluppare e rafforzare la probabilità di sopravvivenza del complessivo contesto territoriale. Lazione dell'Organo di Governo deve tendere a sviluppare l'interazione tra le componenti sistemiche, allo scopo di far emergere caratteri distintivi del sistema. Tale passaggio è funzionale alla valutazione delle volontà degli attori di contesto e alla finalità ultima di generare un aumento di consonanza e, quindi, un effetto di risonanza. I simboli utilizzati indicano la necessità di instaurare un adeguato flusso comunicativo, riguardante gli aspetti che compongono un punto di interesse (Point Of Interest - POI) preso in esame, fra l'Organo di Governo territoriale e le varietà di aspettative presenti nel contesto di riferimento. Tali aspettative, in particolare, sono 
rappresentate dapprima dalla estrapolazione delle opinioni degli utenti (livello "data") e, successivamente, dall'elaborazione delle stesse attraverso la metodologia dell'ABSA (livello "information”).

Fig. 7: Il processo decisionale territoriale tra estrapolazione dei dati e elaborazione delle informazioni

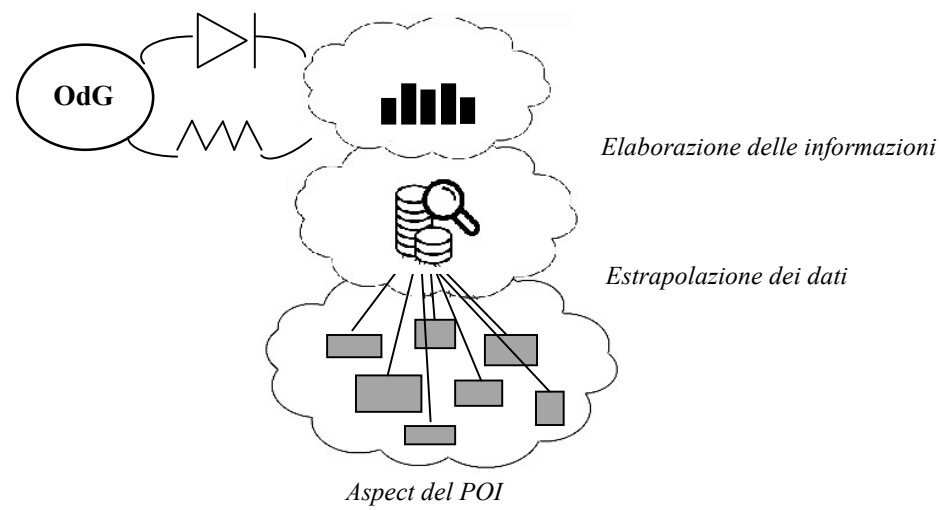

Fonte: elaborazione degli autori

Tramite il trattamento computazionale dell'opinione pubblica (Pang and Lee, 2008; Wang et al., 2012), dunque, è possibile rendere il processo decisionale nel e del sistema vitale territorio indirizzato alla valorizzazione delle potenzialità e delle vocazioni riferibili all'area con ricadute positive in termini di opportunità, benessere, qualità della vita, ecc. per l'insieme degli attori interessati. Questi ultimi esprimono precise attese di valore nei confronti del sistema osservato, sia esso l'intero territorio, il singolo punto di interesse o i diversi aspetti che lo compongono, definendo i relativi obiettivi di efficacia. Nel caso particolare, emergono dall'analisi aspetti quali la riduzione della criminalità e l'ottimizzazione della gestione delle risorse, che diventano nuovi obiettivi della governance locale, e spingono, in maniera puntuale, a compiere interventi tempestivi volti alla loro risoluzione.

\section{Implicazioni teoriche e manageriali}

Il lavoro proposto offre interessanti implicazioni teoriche, combinando, in un unico modello, l'Approccio Sistemico Vitale (Golinelli, 2002, 2005; Barile, 2009b; Barile et al., 2015), lente interpretativa della realtà, con l'ABSA (Liu, 2012), tecnica di estrazione e valutazione di opinioni riguardo entità specifiche. Il fine è quello di integrare ad un corpus teorico, in maniera innovativa, tecniche di analisi testuale e definire un modello che consenta non solo la raccolta e l'analisi dei dati, ma anche -e soprattuttoun approccio interpretativo alle informazioni ottenute.

Inoltre, l'approccio sistemico, combinato alla tecnica di analisi ed elaborazione del testo proposta, offre forti spunti di rimando ai processi di
Sergio Barile

Irene Fulco

Francesca Loia

Approccio sistemico vitale e aspect based sentiment e aspect based sentiment
analysis per il governo del territorio 
sinergie Vol. 37, Issue 1, 2019

co-creazione di valore (Barile e Polese, 2010). L'ASV, infatti, riconoscendo la complessità dei fenomeni sociali, in generale, e dei processi decisionali territoriali, nello specifico, spinge a valutare numerose informazioni, senza perdere una visione sistemica, al fine di co-creare valore attraverso una sempre più dinamica interazione con gli attori di contesto (Barile et al., 2012b). Il lavoro proposto, dunque, combinando un approccio analitico, che spinge a porre attenzione alle singole parti (Popovič et al., 2012), con un approccio olistico, privilegia una prospettiva relazionale e suggerisce una metodologia innovativa in base alla quale prevalgono le dinamiche del sistema rispetto alle componenti strutturali, al fine di favorire un continuo processo di creazione di valore (Barile e Saviano, 2014).

L'elaborato, ancora, evidenzia il nesso sempre più forte ed inscindibile, che si sta creando tra tecnologia, persone e istituzioni, lanciando le basi di un concetto di città sempre più intelligente, fondato su una serie di componenti multidimensionali quali fattori chiave per un'iniziativa smart city di successo (Nam e Pardo, 2011). L'integrazione delle infrastrutture, i servizi mediati dalla tecnologia, l'apprendimento sociale per il rafforzamento delle infrastrutture umane, i sistemi di governance per il miglioramento istituzionale e l'impegno dei cittadini sono solo alcune delle componenti su cui fondare i nuovi sistemi di governance delle città moderne (Nam e Pardo, 2011; Barile et al., 2017; Pellicano et al., 2019).

Da un punto di vista manageriale, il modello si presenta come un valido supporto alle attività decisionali dell'Organo di Governo territoriale, proponendo, in maniera flessibile, valutazioni condivise sui livelli di "sentiment" percepiti dalla comunità rispetto all'area urbana di interesse. E possibile, pertanto, offrire al decisore un'immediata consapevolezza riguardo alle differenti opinioni espresse dalla community, sia per valutare le principali attrattività di un'area urbana sia per rilevare i sintomi di un principio di malcontento (Medhat et al., 2014). Dunque, a fronte delle informazioni ottenute dai quadri sintetici, l'OdG è in grado di attuare una serie di interventi, volti a stabilire le condizioni per una consonanza di contesto, avendo acquisito una visione d'insieme composta dalle diverse percezioni della collettività (Irvin e Stansbury, 2004; Barile, 2009b).

Con la crescente disponibilità e popolarità di opinioni nei siti di recensioni online e blog personali, infatti, si aprono nuove opportunità e sfide per il decision-making, che trovano nel trattamento computazionale delle opinioni presenti sul web la strada da percorrere per sviluppare sistemi in grado di poter rappresentare lopinione pubblica su diversi aspetti di interesse (Pang and Lee, 2008). I social network e le community online, dunque, spingono alla collaborazione tra gli utenti e, attraverso le tecnologie di social computing (Wang et al., 2007), non contribuiscono solamente ad offrire potenziali informazioni, ma conducono sempre di più allemersione del concetto di collective intelligence, un'intelligenza distribuita che crea sinergia tra i diversi attori che interagiscono (Lévy, 2010).

Gli strumenti disponibili per gestire il volume, la velocità e la varietà dei Big data, inoltre, migliorano repentinamente e richiedono nuove abilità, al fine di gestire questa nuova sfida high-tech (McAfee et al., 2012). La figura del manager del futuro, dunque, fortemente connessa agli sviluppi 
tecnologici, dovrà mirare allo sviluppo di capacità trasversali adeguate a fronteggiare il mutevole e dinamico contesto sociale e tecnologico (Barile et al., 2013b; Loia e Fulco, 2018).

Infine, i risultati di tale lavoro possono essere estesi a diversi ambiti, oltre a quello territoriale. Ad esempio, potrebbero offrire interessanti spunti di riflessione per quanto riguarda il processo di sviluppo di un prodotto, consentendo di valutare la percezione dell'utente in merito a caratteristiche specifiche dello stesso, tramite unanalisi delle recensioni e dei feedback lasciati su community on-line. La consapevolezza della percezione negativa può guidare nell'identificazione di difetti latenti che, una volta superati, possono condurre allo sviluppo di nuovi prodotti più performanti e vicini ai desideri del consumatore (Rowley et al., 2007).

\section{Originalità del lavoro, future linee di ricerca e conclusioni}

Loriginalità del lavoro risiede, dunque, nel tentativo di integrare, per la prima volta, al framework concettuale dell'approccio sistemico vitale, già in precedenza impiegato per interpretare problematiche legate al territorio (Barile e Golinelli, 2008; Polese, 2005; Barile et al., 2013a), l'impianto metodologico dell'Aspect Based Sentiment Analysis, per portare avanti ricerche di tipo non solo qualitativo, come già effettuato nei precedenti lavori, ma anche quantitativo, al fine di garantire una maggiore accuratezza nell'analisi dei risultati. Infatti, i due approcci sono considerati complementari (Firestone, 1987) e permettono, da un lato, di ottenere dati strutturati dai quali è possibile trarre analisi di vario tipo e, dall'altro, di applicare un approccio interpretativo e decisionale alla moltitudine di informazioni ricavate.

Tra i futuri sviluppi dello studio, si prevede la possibilità di estendere le logiche delineate nel lavoro, compiendo un'analisi non più sui singoli punti ma su un'area urbana di interesse, determinata, a sua volta, dall'aggregazione degli asset in essa presenti. Il fine è quello di fornire in output informazioni che consentano all'Organo di Governo di implementare azioni volte ad un maggior coordinamento delle componenti coinvolte nello sviluppo dello specifico sistema territoriale. Inoltre, si prevede di affinare il modello proposto, ricercando funzioni di aggregazione per le tecniche di ABSA più sofisticate, per compiere analisi territoriali con maggiore precisione. Sarebbe, infine, di grande interesse integrare al modello una possibile valutazione dei livelli di consonanza e risonanza ottenuti, al fine di fornire all'Organo di Governo territoriale un'informazione sull'efficacia delle politiche territoriali intraprese, in un circolo virtuoso mirato al perseguimento di una maggiore probabilità di sopravvivenza nel contesto considerato.

Concludendo, la rilettura in chiave sistemico vitale, affiancata da tecniche quali la ABSA, che contribuiscono ad accrescere la consapevolezza di un irriducibile nesso che lega ogni entità in un'unica grande rete virale (Capra, 1997), nasce dall'esigenza di un profondo ripensamento nell'approccio di analisi e di conseguente risoluzione delle problematiche legate al governo di qualsiasi sistema, soprattutto se si fa
Sergio Barile

Irene Fulco

Francesca Loia

etro Vito

Approccio sistemico vitale

e aspect based sentiment

analysis per il governo del territorio 
sinergie Vol. 37, Issue 1, 2019

riferimento ad un'entità multi-stakeholder e multi-dimensionale quale il sistema territorio. La varietà di aspettative, prospettive e interessi presenti nel contesto di riferimento è origine dei tipici problemi legati al governo del territorio. In questa direzione, la proposta di un modello di supporto al processo decisionale dell'Organo di Governo territoriale, che abbia alla base la concezione del territorio come sistema vitale, consente il superamento dell'ottica tradizionale, attenta esclusivamente alla fisicità delle sue componenti strutturali, spostando l'accento sull'interazione tra le componenti sistemiche e sui concetti chiave di 'consonanza', 'risonanza' e 'rilevanza', centrali nel processo decisionale dell'Organo di Governo, in una più ampia e generale visione di 'vitalità sistemica'.

\section{Bibliografia}

ARGIOLAS G., CABRAS S., DESSI C., FLORIS M. (2009), "Building innovative models of territorial governance", Journal of Place Management and Development, vol. 2, n. 3, pp. 178-210.

BACCIANELLA S., ESULI A., SEBASTIANI F. (2010), "Sentiwordnet 3.0: an enhanced lexical resource for sentiment analysis and opinion mining", LREC, vol. 10, n. 2010, pp. 2200-2204.

BARILE S. (2009a), Management sistemico vitale, Giappichelli, Torino.

BARILE S., POLESE F. (2010), "Linking the viable system and many-to-many network approaches to service-dominant logic and service science", International Journal of Quality and Service Sciences, vol. 2, n. 1, pp. 23-42.

BARILE S. (2011), "L'approccio sistemico vitale per lo sviluppo del territorio", Sinergie, n. 84, pp. 47-87.

BARILE S., CALABRESE M. (2011), "Business design e consonanza di contesto", Sinergie rapporti di ricerca, n. 32.

BARILE S., DI NAUTA P. (2011), "Viable Systems Approach for territory development", in Various Authors, Contributions to theoretical and practical advances in management - A Viable Systems Approach (VSA), International Printing, Avellino, pp. 199-243

BARILE S., GOLINELLI C.M. (2008), "Modalità e limiti dell'azione di governo del territorio in ottica sistemica", in Barile S. (a cura di), L'impresa come sistema. Contributi sull'Approccio Sistemico Vitale (ASV), Giappichelli, Torino, pp. 243-268.

BARILE S., GOLINELli G.M., MONTELLA M., SAVIANO M. (2012a), “A systems view of cultural heritage. The case of landscape", in Morvillo A. (ed.), Advances in Tourism Studies. In memory of Clara S. Petrillo, Collana "Services and Competitiveness", McGraw-Hill Education, New York, pp. 361-379.

BARILE S., PELS J., POLESE F., SAVIANO M. (2012b), "An introduction to the viable systems approach and its contribution to marketing”, Journal of Business Market Management, vol. 5, n. 2, pp. 54-78.

BARILE S., QUATTROCIOCCHI B., CALABRESE M., IANDOLO F. (2018), "Sustainability and the Viable Systems Approach: Opportunities and Issues for the Governance of the Territory", Sustainability, vol. 10, n. 3, pp. 790.

BARILE S., SANCETTA G., SAVIANO M. (2015), Management. Il modello sistemico e le decisioni manageriali, vol. I, Giappichelli, Torino. 
BARILE S., SAVIANO M. (2008), "Le basi del pensiero sistemico: la dicotomia struttura sistema", in Barile S. (a cura di), L'impresa come sistema, Giappichelli, Torino.

BARILE S., SAVIANO M. (2011), "Foundations of systems thinking: the structuresystem paradigm", in Various Authors, Contributions to theoretical and practical advances in management. A Viable Systems Approach (VSA), International Printing, Avellino, pp. 1-26.

BARILE S., SAVIANO M. (2012), "Dalla gestione dei beni culturali al governo del sistema dei beni culturali", in Golinelli G.M. (a cura di), Patrimonio culturale e creazione di valore. Verso nuovi percorsi, Cedam, Padova, pp. 97-148.

BARILE S., SAVIANO M. (2014), "Resource integration and value co-creation in cultural heritage management", in Handbook of Research on Management of Cultural Products: E-Relationship Marketing and Accessibility Perspectives (pp. 58-82), IGI Global.

BARILE S., SAVIANO M., IANDOLO F., CALABRESE M. (2014), "The viable systems approach and its contribution to the analysis of sustainable business behaviors", Systems Research and Behavioral Science, vol. 31, n. 6, pp. 683-695.

BARILE S., SAVIANO M., POLESE F., DI NAUTA P. (2013a), "Il rapporto impresa-territorio tra efficienza locale, efficacia di contesto e sostenibilità ambientale", Sinergie, n. 90, pp. 25-49.

BARILE S., CIASULLO M.V., TROISI O., SARNO D. (2017), "The role of technology and institutions in tourism service ecosystems: Findings from a case study", The TQM Journal, vol. 29, n. 6, pp. 811-833.

BARILE S. (2009b), "The dynamic of informative varieties in the processes of decision making", Proceedings of the $13^{\text {th }}$ World Multi-Conference on Systemics, Cybernetics and Informatics: WMSCI, Florida.

BARILE S., CALABRESE M., POLESE F., SAVIANO M. (2013b), "Il governo dei sistemi complessi tra competenze attuali e capacità potenziali”, Barile S., Eletti V., Matteuzzi M.(a cura di), Decisioni e scelte in contesti complessi, Cedam-Kluwer, Lavis.

BARON R.A., HMIELESKI K.M., HENRY R.A. (2012), “Entrepreneurs' dispositional positive affect: The potential benefits-and potential costs-of being "up"', Journal of Business Venturing, vol. 27, n. 3, pp. 310-324.

BASILE G., DOMINICI G., TANI M. (2016), "Place Marketing and Management: A Complex Adaptive Systems View. The Strategic Planning of the City of Avellino, Italy", Systemic Practice and Action Research, vol. 29, n. 5, pp. 469-484.

BEER S. (1972), Brain of the firm; a development in management cybernetics, Herder and Herder, New York.

BEN-AMAR W., FRANCOEUR C., HAFSI T., LABELLE R. (2013), "What makes better boards? A closer look at diversity and ownership", British Journal of Management, vol. 24, n. 1, pp. 85-101.

BUHALIS D. (2000), "Marketing the competitive destination of the future", Tourism Management, vol. 21, n. 1, pp. 97-116.

CALABI D., INDOVINA F. (1973), "Sull'uso capitalistico del territorio", Archivio di studi urbani e regionali, vol. 4, n. 2, pp. 3-20.

CAPRA F. (1997), The web of life: A new scientific understanding of living systems, Anchor, New York. 
CARRUBBO L. (2013), La Co-creazione di valore nelle destinazioni turistiche, Rirea, Roma.

CERTOMA C., CORSINI F., RIZZI F. (2015), “Crowdsourcing urban sustainability. Data, people and technologies in participatory governance”, Futures, n. 74, pp. 93-106.

CIASUlLO M.V., POLESE F., TROISI O., CARRUBBO L. (2016), "How Service Innovation Contributes to Co-Create Value in Service Networks", in Borangiu T., Dragoicea M., Novoa H. (Eds.), Exploring Services Science (vol. 247, pp. 170-183), Berlin: Springer-Verlag Berlin.

CROSTA P.L. (2000), "Società e territorio, al plurale. Lo spazio pubblico-quale bene pubblico-come esito eventuale dell'interazione sociale", Foedus, n. 1, pp. 40-53.

CROUCH G.I., RITCHIE J.B. (1999), “Tourism, competitiveness, and societal prosperity", Journal of Business Research, vol. 44, n. 3, pp. 137-152.

D'ANIELLO G., GAETA M., LOIA F., REFORMAT M., TOTI D. (2018), “An Environment for Collective Perception based on Fuzzy and Semantic Approaches", Journal of Artificial Intelligence and Soft Computing Research, vol. 8, n. 3, pp. 191-210

DE JONG A., SONG M., SONG L.Z. (2013), "How lead founder personality affects new venture performance: The mediating role of team conflict", Journal of Management, vol. 39, n. 7, pp. 1825-1854.

DE WIT F.R.C., GREER L.L., JEHN K.A. (2012), “The Paradox of Intragroup Conflict: A Meta-Analysis”, Journal of Applied Psychology, vol. 97, n. 2, pp. 360-390.

DECHURCH L.A., MESMER-MAGNUS J.R., DOTY D. (2013), "Moving beyond relationship and task conflict: Toward a process-state perspective", Journal of Applied Psychology, vol. 98, n. 4, pp. 559.

DELLA CORTE V. (2000), La gestione dei sistemi locali di offerta turistica, Cedam, Padova.

DENECKE K. (2008, April), "Using sentiwordnet for multilingual sentiment analysis", in Data Engineering Workshop, 2008. ICDEW 2008. IEEE 24th International Conference on (pp. 507-512), IEEE.

EHLINGER S., PERRET V., CHABAUD D. (2007), "Quelle gouvernance pour les réseaux territorialisés d’organisations?”, Revue Francaise de Gestion, vol. 170, n. 1, pp. 155-171.

EISENHARDT K.M., ZBARACKI M.J. (1992), "Strategic decision making", Strategic Management Journal, vol. 13, n. S2, pp. 17-37.

ESULI A., SEBASTIANI F. (2007), "SentiWordNet: a high-coverage lexical resource for opinion mining”, Evaluation, n. 17, pp. 1-26.

FIRESTONE W.A. (1987), "Meaning in method: The rhetoric of quantitative and qualitative research", Educational researcher, vol. 16, n. 7, pp. 16-21.

FOTINO F., CALABRESE M., LETTIERI M. (2018), "Co-creating value in urban public policy contexts: A different approach", Land Use Policy, vol. 79, pp. 20-29.

FOUCAULT, M. (1994), Eterotopia: luoghi e non-luoghi metropolitani (Vol. 2), Mimesis, Sesto San Giovanni.

FRANCH M. (2002), Destination management. Governare il turismo tra locale e globale, Giappichelli, Torino.

GALTON F. (1907), "Vox populi (The wisdom of crowds)", Nature, vol. 75, n. 7, pp. 450-451. 
GAVETTI G., GREVE H.R., LEVINTHAL D.A., OCASIO W. (2012), “The behavioral theory of the firm: Assessment and prospects", The academy of management annals, vol. 6, n. 1, pp. 1-40.

GOLINELLI G.M. (2002), L’approccio sistemico al governo dell'impresa. Verso la scientificazione dellazione di governo II, Cedam, Padova.

GOLINELLI G.M. (2005), Lapproccio sistemico al governo dell'impresa. L'impresa sistema vitale, I, Cedam, Padova.

GOLINELLI G.M. (2011), L'Approccio Sistemico Vitale (ASV) al governo dell'impresa. Verso la scientificazione dellazione di governo, Cedam, Padova.

IANDOLO F., FULCO I., CARRUBBO L., ARMENIA S. (2016), "Destination mobility management in the light of service research: the "good practices" of South Tyrol", Esperienze d'Impresa, vol. 24, n. 2, pp. 39-63.

IANDOLO F., FULCO I., BASSANO C., D’AMORE R. (2019), "Managing a tourism destination as a viable complex system. The case of Arbatax Park", Land Use Policy, vol. 84, pp. 21-30.

INTEZARI A., GRESSEL S. (2017), "Information and reformation in KM systems: big data and strategic decision-making", Journal of Knowledge Management, vol. 21, n. 1, pp. 71-91.

IRVIN R.A., STANSBURY J. (2004), "Citizen participation in decision making: is it worth the effort?", Public Administration Review, vol. 64, n. 1, pp. 55-65.

KARAHANNA E., PRESTON D.S. (2013), "The Effect of Social Capital of the Relationship Between the CIO and Top Management Team on Firm Performance", Journal of Management Information Systems, vol. 30, n. 1, pp. 15-56.

LEFEBVRE H. (1974), "La production de l'espace", L'Homme et la société, vol. 31, n. 1 , pp. 15-32.

LÉVY P. (2010), "From social computing to reflexive collective intelligence: The IEML research program", Information Sciences, vol. 180, n. 1, pp. 71-94.

LI Y., HOU M., LIU H., LIU Y. (2012), “Towards a theoretical framework of strategic decision, supporting capability and information sharing under the context of Internet of Things", Information Technology and Management, vol. 13, $\mathrm{n}$. 4, pp. 205-216.

LIU B. (2012), "Sentiment analysis and opinion mining", Synthesis lectures on human language technologies, vol. 5, n. 1, pp. 1-167.

LOIA F., FULCO I. (2018), "The effect of cross-functional collaboration on technology commercialization performance: the mediating role of knowledge creation and organizational resilience", in Evoluzionismo sistemico: il fascino della precarietà, (Eds.) Paniccia P.M.A., Barile S., Aracne (2018), pp. 93-99.

MANYIKA J., CHUI M., BROWN B., BUGHIN J., DOBBS R., ROXBURGH C., BYERS A.H. (2011), Big data: The next frontier for innovation, competition, and productivity, Disponibile sul sito https://www. mckinsey.com/business-functions/digital-mckinsey/our-insights/ big-data-the-next-frontier-for-innovation.

MARINO V., GALLUCCI C., BELLELLI P. (2013), "The conceptual framework of Placescape. A new Service Marketing perspective for the territory system", in Gummesson E., Mele C., Polese F. (Eds.), Service Dominant Logic, Network and Systems Theory and Service Science: Integrating three Perspectives for a New Service Agenda, Giannini, Napoli.
Sergio Barile

Irene Fulco

rancesca Loia

Approccio sistemico vitale aspect based sentiment analysis per il governo del territorio 
MARTINI U. (2002), "Da luoghi a destinazioni turistiche. Ipotesi di destination management nel turismo alpino", in Franch M. (Ed.), Destination management. Governare il turismo tra locale e globale, pp. 107-111. Giappichelli, Torino.

MATTINA, C. (2008), "Governing "local democracy" at the urban level: Neighborhood committees in Marseille, Toulon and Nice", Sociologie du Travail, vol. 50, n. 2, pp. 184-199.

MCAFEE A., BRYNJOLFSSON E., DAVENPORT T.H., PATIL D.J., BARTON D. (2012), "Big data: the management revolution", Harvard Business Review, vol. 90 , n. 10, pp. 60-68.

MEDHAT W., HASSAN A., KORASHY H. (2014), "Sentiment analysis algorithms and applications: A survey", Ain Shams Engineering Journal, vol. 5, n. 4, pp. 1093-1113.

NAM T., PARDO T.A. (2011, June), "Conceptualizing smart city with dimensions of technology, people, and institutions", in Proceedings of the $12^{\text {th }}$ annual international digital government research conference: digital government innovation in challenging times (pp. 282-291), ACM.

NAPOLITANO M.R. (2000), Dal Marketing Territoriale alla Gestione Competitiva del Territorio Edizioni scientifiche italiane, Napoli.

PAK A., PAROUBEK P. (2010), “Twitter as a corpus for sentiment analysis and opinion mining”, $L R E C$, vol. 10, n. 2010.

PANATI G., GOLINELLI G. (1988), Tecnica economica industriale e commerciale: imprese, strategie e management, Nuova Italia Scientifica, Roma.

PANG B., LEE L. (2008), "Opinion mining and sentiment analysis", Foundations and Trends in Information Retrieval, vol. 2, n. 1-2, pp. 1-135.

PAVLOPOULOS I. (2014), Aspect Based Sentiment Analysis, Athens University of Economics and Business.

PELliCANO M., MAIONE G., LOIA F., CALABRESE M. (2019), "Value CoCreation Practices in Smart city Ecosystem", Journal of Service Science and Management, vol. 12, n. 1, pp. 34-57.

PENCARELLI T., FORLANI F. (2003), “'Il marketing dei distretti turistici-sistemi vitali nell'economia delle esperienze" (versione ampliata).

PILOTTI L., APA R., TEDESCHI-TOSCHI A., SARMAN I. (2013), "La competitività dinamica degli ecosistemi territoriali. Il caso degli ecosistemi turistici multilocali tra Italia e Svizzera", Sinergie, n.91, pp. 83-102.

PILOTTI L., TEDESCHI-TOSCHI A. (2011), "La competitività ecologica dei sistemi territoriali tra identità, immagine e vantaggi "costruiti", Quale marketing dei servizi turistici di fronte alla "long tail"?", International Marketing Trends Conference, Paris, 20th-22nd January 2011

POLESE F. (2002), "Lapproccio sistemico vitale per l'analisi del territorio: Il caso del Parco Nazionale del Vesuvio", Esperienze d'Impresa, vol. X, n. 2, pp. 121-139.

POLESE F. (2005), "Risorse e identità locale per la valorizzazione del territorio: un percorso difficile di integrazione sistemica e networking", in Morvillo A., Petrillo C. (a cura di), Sviluppo del territorio: Identità e integrazione - Il caso del Parco Nazionale del Cilento e del Vallo di Diano, Collana IRAT - CNR, pp.103-194.

POLESE F., CARRUBBO L., BRUNI R., MAIONE G. (2017), “The viable system perspective of actors in eco-systems", TQM Journal, vol. 29, n. 6, pp. 783-799. 
POLESE F., MINGUZZI A. (2009), "Networking approaches for sustainable destination management", Advances in Tourism Destination Marketing: Managing Networks, n. 113, pp. 113-124.

POPOVIČ A., HACKNEY R., COELHO P.S., JAKLIČ J. (2012), “Towards business intelligence systems success: Effects of maturity and culture on analytical decision making", Decision Support Systems, vol. 54, n. 1, pp. 729-739.

POST C., BYRON K. (2014), "Women on Boards and Firm Financial Performance: A Meta-Analysis", Academy of Management Journal, vol. 58, n. 5, pp. 1546-1571.

POST C., RAHMAN N., MCQUILLEN C. (2015), "From board composition to corporate environmental performance through sustainability-themed alliances", Journal of Business Ethics, vol. 130, n. 2, pp. 423-435.

PRÉTECEILLE, E. (1974), "La planification urbaine: les contradictions de l'urbanisation capitaliste", Economie et Politique, n. 236, pp. 94-114.

PROVOST F., FAWCETT T. (2013), "Data science and its relationship to big data and data-driven decision making", Big Data, vol. 1, n. 1, pp. 51-59.

RISPOLI M., TAMMA M. (1995), Risposte strategiche alla complessità: le forme di offerta dei prodotti alberghieri, Giappichelli, Torino.

ROWLEY J., KUPIEC-TEAHAN B., LEEMING E. (2007), “Customer community and co-creation: a case study", Marketing Intelligence and Planning, vol. 25, n. 2, pp. 136-146

SAMUEL K.E., CARRE M. (2018), "Entre communautés de pratique et communautés épistémiques: L'émergence de communautés hybrides dans les espaces urbains", Systemes d'Information et Management, vol. 23, n. 1, pp. 41-63.

SCHILLACI C.E., GATTI C. (2010), "E pluribus unum: intenzionalità collettiva e governo dei sistemi territoriali”, Sinergie, n. 84, pp. 21-45.

SCIARELLI S. (2007), Il management dei sistemi turistici locali: strategie e strumenti per la governance, Giappichelli, Torino.

SCOTT A., STORPER M. (2003), "Regions, globalization, development", Regional studies, vol. 37, n. 6-7, pp. 579-593.

SHEPHERD N.G., RUDD J.M. (2014), "The Influence of Context on the Strategic Decision-making Process: A Review of the Literature”, International Journal of Management Reviews, vol. 16, n. 3, pp. 340-364.

SIMON H.A. (1957), Models of man: social and rational; mathematical essays on rational human behavior in society setting, Wiley.

SIMONE C., BARILE S., CALABRESE M. (2018), "Managing territory and its complexity: a decision-making model based on the viable system approach (VSA)", Land Use Policy, vol. 72, pp. 493-502.

SUROWIECKI J. (2007), La saggezza della folla, Fusi orari, Roma.

TAMMA M. (2000), “Aspetti strategici del destination management", in Pechlaner e Weiermair K. (a cura di), Destination Management, Touring Univeristy Press, T.C.I..

TOLEDO PARRA C.A., GAMBOA SARMIENTO S.C., DI FATTA D. (2017), "Studying University as Social Systems Using the Viable System Model: mApp and Semantic Web Technologies at the Industrial University of Santander", Journal of Organisational Transformation and Social Change, vol. 14, n. 1, pp. 56-77.

TROISI O., D'ARCO M., LOIA F., MAIONE G. (2018), "Big data management: The case of Mulino Bianco's engagement platform for value co-creation", International Journal of Engineering Business Management, n. 10, pp. 1-8. 
VAN ECK N.J., WALTMAN L. (2014), "Visualizing bibliometric networks", in Measuring scholarly impact, pp. 285-320: Springer.

VELOTTI L., BOTTI A., VESCI M. (2012), "Public-private partnerships and network governance What Are the Challenges?", Public Performance and Management Review, vol. 36, n. 2, pp. 340-365.

WALKER M. (2017), “The Search for Viability: A practitioner's view of how the Viable Systems Model is helping transform English local government (and why it has passed unrecognised)", Systems Research and Behavioral Science, vol. 34, n. 3, pp. 313-334.

WANG F.Y., CARLEY K.M., ZENG D., MAO W. (2007), "Social computing: From social informatics to social intelligence", IEEE Intelligent systems, vol. $22, \mathrm{n}$. 2, pp. 79-83.

WANG H., CAN D., KAZEMZADEH A., BAR F., NARAYANAN S. (2012, July), "A system for real-time twitter sentiment analysis of 2012 us presidential election cycle", in Proceedings of the ACL 2012 System Demonstrations (pp. 115-120), Association for Computational Linguistics.

WILSON T., WIEBE J., HOFFMANN P. (2005), "Recognizing contextual polarity in phrase-level sentiment analysis", in Proceedings of the conference on human language technology and empirical methods in natural language processing (pp. 347-354), Association for Computational Linguistics.

\section{Sitografia}

http://sinergiejournal.eu/

https://web.archive.org/web/20130120065922/http://www.traveltradejournal.com/ newsdetails.php?nid=353

https://www.tripadvisor.it/Attraction_Review -g187791-d190131-Review s-Trevi_ Fountain-Rome_Lazio.html

\section{Academic or professional position and contacts}

\section{Sergio Barile}

Full Professor of Management

Sapienza University of Roma - Italy

e-mail: sergio.barile@uniromal.it

Irene Fulco

Phd in Management

Sapienza University of Roma - Italy e-mail: irene.fulco@uniroma1.it

sinergie
italian journal of management

ISSN 0393-5108 DOI $10.7433 / \mathrm{s} 108.2019 .10$
pp. $171-196$

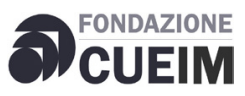

\section{S I}

Società Italiana di MANAGEMENT
Francesca Loia

Phd in Management

Sapienza University of Roma - Italy

e-mail: francesca.loia@uniromal.it

\section{Pietro Vito}

Phd in Management

Sapienza University of Roma - Italy e-mail: pietro.vito@uniroma1.it 